\title{
Markt und Magie, Kommunikation und Kult Zum Kino als Ereignisraum
}

von Reinhold Jacobi

Das Schönste im Kino ist das Wunderbare (Kurt Pinthus, 1913) ${ }^{1}$

1.

Bei den Feiern, mit denen der Film anläßlich seines 1995 erreichten Alters von 100 Jahren gewürdigt wird, steht das technische und künstlerische Produkt als Einzelwerk wie als gattungsbildendes Medium im Vordergrund des Interesses. Die Filmkritik, die Filmpublizistik allgemein rekapituliert in vielfältiger Weise die Leistungen des Films: Welche Filme hat es gegeben, welchen Wert hatten sie - künstlerisch wie wirtschaftlich -, aus welchem Grunde hatten sie Bestand? Man würdigt berechtigterweise den Film als das Medium, dessen erste Lebenshälfte durch eigene Aufführungshäuser, die Kinos, bestimmt war. Nach diesem Abschnitt taten sich durch die Entwicklung des Fernsehens und der Videotechnik zwei weitere Verwertungsschienen auf, deren Ambivalenz die beiden letzten Jahrzehnte des film- und fernsehpolitischen Diskurses geprägt hat. ${ }^{2}$ Nunmehr steht eine weitere technische Entwicklung an, mit deren Hilfe der Kinofilm über CD-ROM und diverse PC-Weitungen anderer Art eine noch weitere Verbreitung finden wird.

Die Wirkungsentwicklung des Films quer durch die sich verändernde Nutzungslandschaft wird 1995 ebenfalls erinnert. Dabei wird mit Recht auf die Ambivalenz der Filmnutzung als Einzelfilm in unterschiedlichem Kontext wie als Massenkonsumgut vor allem der wuchernden Fernsehprogramme hinzuweisen sein: Der Kinofilm als Einzelwerk, in sich geschlossen und autonom mit seiner Erzählgeschichte, wird seit zwei Jahrzehnten zunehmend nicht nur beliebig häufig und unterschiedslos im programmsüchtigen Fernsehen zweit- und drittver-

Dr. Reinhold Jacobi ist Leiter der ,Zentralstelle Medien' der Deutschen Bischofskonferenz in Bonn.

1 Kurt Pinthus (Hg.), Das Kinobuch, Zürich 1963 (= Dokumentarische Neuausgabe des "Kinobuchs" von 1913/14), 27.

2 Vgl. dazu die Daten zur Medienentwicklung der letzten Jahre v.a. in den jährlichen „Filmstatistischen Taschenbüchern der Spitzenorganisation der Filmwirtschaft (Wiesbaden)" sowie die ebenfalls jährlichen „Daten zur Mediensituation in Deutschland" (1993 und früher) (= Sonderdrucke „Media Perspektiven" der Arbeitsgemeinschaft der ARD-Werbegesellschaften). 
wertet; er hat sich darüber hinaus durch die Serie bis hin zur "daily soap" veralltäglicht und in schiere Wahrnehmungsbeliebigkeit vulgarisiert. Dennoch werden permanent aufwendige und immer wieder auch künstlerisch ambitionierte Kinofilme hergestellt, die als Einzelprodukte die Wahrnehmung lohnen, da sie - ob man will oder nicht, ob man dies realisiert oder nicht - außerordentlich intensive Beteiligte sind am ständigen Fortschreiben des Zeitbewußtseins. Der Film ist aufgrund seiner außergewöhnlichen Fähigkeiten und Eigenschaften zum spezifischsten Ausdrucksmittel, zum markantesten Medium dieses ausgehenden Jahrhunderts geworden. Inwiefern dies nicht pauschale Behauptung bleiben darf, soll eine (kurze) Würdigung weniger des einzelnen Films als des Nutzungsraums Kino zeigen, der nach wie vor aus den verschiedensten Gründen der eigentliche und wichtigste Ort filmischer Wahrnehmung ist. Was fand und findet dort statt?

\section{I.}

2.

Nach der Erfindung der Cinematographie in den 90er Jahren des 19. Jahrhunderts war der Film zunächst ein Teil des Jahrmarkts, des Varietés und Rummels; seine sensationelle Fähigkeit, mit bewegten Bildern Wirklichkeit und Phantasie abzubilden, hatte etwas Marktschreierisches. Das Verständnismittel Film, noch stumm, bedurfte keiner Sprache, um seine Absicht auszudrücken. Konsequenterweise nutzten ihn zunächst die einfachen Leute, die Arbeiter, in den USA vor dem 1. Weltkrieg vor allem Einwanderer, die der englischen Sprache (noch) nicht mächtig waren, und zwar in den sog. "Nickelodeons". ${ }^{3}$ Auch in Europa reagierten Unternehmen auf das Interesse gerade bei Arbeitern durch bevorzugten Kinobau in Arbeitervierteln und Arbeiterstädten.4 "Vor 1914 spiegelte sich im Film eindeutig der Geschmack der Unterschichten. ${ }^{\text {5 }}$

Salonfähigkeit erreichte das Kino gegen Ende des 2. Jahrzehnts, als die Filmlänge allmählich das heutige, sog. „abendfüllende “ Format von 60 bis 90 Minuten erreicht hatte: Man konnte ins Kino mit ähnlicher unterhaltsamer oder kulturbeflissener Absicht gehen wie ins Theater. Die Analogie für das Bürgertum ergab sich nicht zuletzt auch durch die immer erstaunlicheren schauspielerischen Leistungen, zu denen thematische Aspekte hinzukamen. Die vorhandene und die vorstellbare Wirklichkeit, Fiktion und Realität in ihrer gesamten Breite fesselten den sich seinem Alltag entziehenden Bürger. Reine Bewegungsstudien wie

3 Dieter Prokop, Soziologie des Films, Darmstadt-Neuwied 1974, 35 u. 64 f.

Ebd., 35.

5 Konrad Lange, Das Kino in Gegenwart und Zukunft (1920), 51 (zit. bei Prokop, 65). 
in den ersten Jahren des Films bis hin zu exotischen Anwendungen der Bewegungstricks etwa durch die phantastische Darstellung einer Mondreise durch Méliès ${ }^{6}$ oder der Einbau von Bewegung in die Handlung des Abenteuerfilms ${ }^{7}$ - in Filmen von nur wenigen Minuten Länge - waren inzwischen durch vollständige Kinoerzählungen abgelöst worden: Robert Wienes expressionistischer Klassiker "Das Kabinett des Dr. Caligari" von 1919, Charlie Chaplins "The Kid" (1920) oder Friedrich Wilhelm Murnaus "Nosferatu - Eine Symphonie des Grauens" (1922) sind abendfüllend. ${ }^{8}$ Die Abkehr von rein abenteuerlichen oder eher exotischen Stoffen wurde schon früh vollzogen: Von den insgesamt ca. 120 Jesus-Verfilmungen aus der gesamten Zeit der Filmgeschichte erschienen vier schon im Jahr 1897. ${ }^{9}$

In den 20er Jahren boomt die Entwicklung des Kinofilms: Er hat seine technischen Möglichkeiten enorm entwickelt und verfeinert, bis ihm Ende des Jahrzehnts als letzter Schritt auch noch die Hinwendung zum Ton gelingt (1927). Vorher hatte er allerdings mit Sergej Eisenstein oder Wsewolod Pudowkin in der UdSSR, mit Charles Chaplin und David W. Griffith in den USA, mit Fritz Lang, Ernst Lubitsch, Georg Wilhelm Pabst oder G. F. Murnau in Deutschland, mit Abel Gance (Napoléon, 1927) und Carl Theodor Dreyer (La Passion de Jeanne d'Arc, 1927) in Frankreich u.a.m. schon Meisterwerke der Filmkunst hervorgebracht.

Der Film diente wegen seiner suggestiven Möglichkeiten schon in den politischen Bewegungen seit dem 1. Weltkrieg immer wieder der Agitation und Propaganda; die frühen Revolutionsjahre der UdSSR erlebten Eisenbahn-Züge, die als rollende Kinosäle durchs Land fuhren ${ }^{10}$; der in Deutschland während der NS-Zeit unter ausschließlicher staatlicher Regie stehende Film brachte nationalsozialistische Ideologie-Ware wie "Hitlerjunge Quex" (1933), "Ohm Krüger" (1941), "Jud Süss" oder "Der ewige Jude" (beide 1940) hervor, daneben aber auch vermeintlich unverfängliche Unterhaltungsfilme mit den Stars des Genres. ${ }^{11}$

6 George Méliès, Die Reise zum Mond, Frankreich 1902 (Le Voyage dans la Lune).

7 Edwin S. Porter, Der große Eisenbahnraub, USA 1903 (The Great Train Robbery).

8 Vgl. Lexikon des Internationalen Films, hg. v. Katholischen Institut für Medieninformation und der Katholischen Filmkommission für Deutschland, 10 Bde., Reinbek 1987.

9 Zwei Filme in Frankreich, einer in Italien, einer in den USA (vgl. Karl-Eugen Hagmann u. Peter Hasenberg (Red.), Jesus in der Hauptrolle. Zur Geschichte und Ästhetik der Jesus-Filme, film-dienst EXTRA, hg. vom Katholischen Institut für Medieninformation in Zusammenarbeit mit der Katholischen Filmkommission für Deutschland 1992, 74).

10 rororo-Filmlexikon, hg. v. Liz-Anne Bawden/Wolfram Tichy, Reinbek 1978 (6 Bde.), Bd. 2, 617ff.

11 rororo-Filmlexikon, Bd. 1., 140: Im Dritten Reich entstanden etwa 1.100 
In den USA wurde die Komödie in ihrer ganzen Bandbreite ausgefaltet. Chaplins Klassiker wie "Moderne Zeiten“ (1936) stand neben den erfolgreichen Serienprodukten mit Stan Laurel und Oliver Hardy (ab 1937), die Filme der chaotischen Marx-Brothers liefen ab 1933, nachdem die 20er Jahre den skurrilen Buster Keaton genossen hatten. Die "Screwball-Comedy", die Meister wie Frank Capra, Ernst Lubitsch und Mitchell Leison zur Blüte brachten, ließen die Folgen der Depression des Jahrzehntwechsels 1920/1930 erträglicher erscheinen, da man sich mit heiter-frivolen Gesellschaftsgeschichten von eigenen Schwierigkeiten ablenken konnte. Nach Kriegsausbruch entdeckten auch die USA und Großbritannien die propagandistischen Möglichkeiten des Films, denen sich aus patriotischer Gesinnung heraus auch und gerade Prominente wie Alfred Hitchcock, John Ford, William Wyler, John Huston oder Frank Capra nicht verweigerten.12 Die 30er und 40er Jahre sind damals vor allem wegen ihrer Ausfaltung verschiedener anderer Genres wie Western (John Ford, Ringo/Stage Coach, 1937), Kriminalfilm John Huston, Die Spur des Falken, 1941), Melodram (Michael Curtiz, Casablanca, 1942; Charles Vidor, Gilda, 1946) und Animationsfilm (Walt Disney, Bambi, 1946) filmkritisch in herausragender Erinnerung. ${ }^{13}$

3.

Vom ehedem von der Wahrnehmung her eher eingeschränkten Interesse an den Anfängen des Kinofilms hatte sich Mitte des Jahrhunderts die Aufmerksamkeit weltweit auf das gesamte, inzwischen genremäßig außerordentlich breite Filmangebot ausgeweitet; für alle Schichten war der Film interessant geworden. Er gehörte nach dem 2. Weltkrieg in allen Ländern, die wirtschaftlich wieder aufgebaut werden und kulturell Schritt fassen mußten - wie vor allem Deutschland mit beiden Teilstaaten und die anderen Staaten Mitteleuropas - zu den bevorzugten Freizeitattraktionen. ${ }^{14}$ Auch die ausgesprochen filmkulturelle Auseinandersetzung in Europa fand nach dem 2. Weltkrieg Möglichkeiten; man hatte Nachholbedarf, die fremden Cinematographien kennen-

Spielfilme, was das Ausmaß der Indoktrination wie die Wertschätzung des Mediums Film bei den Machthabern kennzeichnet.

Vgl. dazu rororo-Filmlexikon, Bd. 2, 522ff, bes. 528f.

13 Vgl. zu den Einzelfilmen Lexikon des Internationalen Films, a.a.O., passim; Peter W. Engelmeier, 100 Jahre Kino. Die großen Filme, Augsburg 1994.

141954 gingen in der Bundesrepublik 736 Mio. Besucher ins Kino, 1955766 Mio., 1956818 Mio., was einen Pro-Kopf-Besuch pro Jahr von 14,4 (1954), 14,8 (1955), 15,6 (1956) ergibt (Filmstatistisches Taschenbuch der Spitzenorganisation der Filmwirtschaft (SPIO), Wiesbaden 1964, 28). In der Folgezeit sank die Anzahl kontinuierlich ab bis auf (1986) 105,7 Mio. (= 1,7 Filmbesuche je Einwohner pro Jahr) (Filmstatistisches Taschenbuch der SPIO 1987, 24). 1992 lagen die Zahlen bei 105,9 Mio. (nach der Wiedervereinigung!) und 1,3 Besuchen je Einwohner pro Jahr (Media Perspektiven, Daten zur Mediensituation in Deutschland 1993, Frankfurt/M. 1993, 67). 
zulernen, die eine total gelenkte Staatskultur konsequent ausgesperrt hatte. ${ }^{15}$ Dieser nicht nur individuell-genießerische Umgang mit dem Film führte zu einer Reihe von Filmfestspiel-Gründungen, wobei Cannes und Locarno 1946 den Anfang machten und Berlin 1951 folgte. Lediglich Venedig hatte schon 1932 - also lange vor dem 2. Weltkrieg ein internationales Festival, das nach dem Krieg im Wettstreit mit den Neugründungen die nunmehr entideologisierte Programmdiskussion auch wieder mit anführte. ${ }^{16}$

Nach dem erwähnten Auf- und Ausbau des Fernsehens ging der Kinobesuch überall zurück; Bequemlichkeit, Wahlfreiheit und die Möglichkeiten der eigenen Filmkopiensammlung auf Video, verbunden mit einer vor der Entwicklung der Videotechnik gelegenen zunehmenden Abstinenz mittlerer und älterer Zuschauerschichten vom Kino wegen der seinerzeitigen Dominanz von Heimat-, Sex- oder EpigonalFilmen des Westerngenres, führten zum Auszug aus dem Kino. Dank einer mit großen Mühen vollzogenen Gegenstrategie vor allem der Filmtheaterwirtschaft hat sich inzwischen der Kinobesuch - hierzulande - auf einer mäßigen Höhe stabilisiert. ${ }^{17}$ Dazu beigetragen haben neben diversen anderen Fakten vor allem der verbesserte Ruf des Kinos wegen größerer Sorgfalt bei Inneneinrichtung und Service, wegen des seit ca. zehn Jahren praktizierten Abbaus der "Schuhkarton"-Mentalität mancher Unternehmer und vor allem wegen der nahezu gänzlichen Abwanderung der Sex- und Porno-Filme, der Horror- und GewaltSchocker aus dem Kino in die Schmuddelecken der Videotheken. Allmählich fand das Publikum mittleren Alters ins Kino zurück, von Fall zu Fall bei renommierten anspruchsvollen Filmen der Problem- wie der Komödienschiene. ${ }^{18}$ Generell allerdings bleibt das Kino ein Ort der Freizeitgestaltung, der Geselligkeit oder des Rückzugs vom Alltag für die dezidiert jüngere Generation der 14- bis 29jährigen.19 Was wird diesen Zuschauern geboten? Was suchen sie im Kino? Was finden sie vor?

15 Gerade in den ersten 15 Jahren nach Kriegsende gab es durch die organisierte Film-Club-Idee in der Bundesrepublik eine sehr ambitionierte filmkulturelle Bewegung.

16 rororo-Filmlexikon, Bd. 1, $209 \mathrm{ff}$.

17 Vgl. Anm. 14.

18 Steven Spielbergs "Schindlers Liste" (USA 1993) oder Sidney Pollacks "Jenseits von Afrika" (USA 1985) auf der einen, Sidney Pollacks "Tootsie“ (USA 1982) oder Doris Dörries „Männer” (Bundesrepublik 1985) auf der anderen Seite.

19 Von denjenigen, die mindestens einmal pro Woche ins Kino gehen $(=2,81$ Mio.) gehören 1,99 Mio. (= $71 \%$ ) dieser Altersgruppe an; weitere $24 \%(=$ 0,66 Mio.) sind 30 bis 49 Jahre alt. Lediglich $5 \%(=0,14$ Mio.) sind älter (Media Perspektiven, a.a.O., 83). 
II.

4.

Die jüngere Generation sieht nichts anderes, als die älteren Mitbürger auch sehen könnten bzw. faktisch sehen. Nur sind letztere schon anders orientiert. Sie haben inzwischen Familie, stehen am Beginn beruflicher Laufbahnen, sind u.U. verbandlich oder politisch engagiert. Ihre Ereignisse werden von anderen Prinzipien und Vorgaben diktiert oder wenigstens geliefert. Allmählich stellen sich bei ihnen für den organisierten Freizeitbereich kulturellen Zuschnitts auch wieder die Paletten traditioneller Erlebnisräume wie Konzertsaal und Theater ein. Hinzugekommen sind die sportlichen Trainingsangebote, die ein Teil der Freizeit absorbieren: Man lebt gesünder bzw. gesundheitsbewußter. Außerdem hat man das Filmangebot des Kinos - wenn man dies denn unbedingt wahrnehmen müßte - mit Zeitverschiebung (von immer geringerem zeitlichen Ausmaß dank Pay-TV z.B.) - im Fernsehen bzw. über Video zur Verfügung.

Angesichts der allgemeinen Zeitbeschleunigung, in der wir leben, ist dies für die Jüngeren allerdings kein Angebot. Sie wollen den neuen Kinofilm sofort. Damit liegen sie im übrigen auf derselben Wellenlänge wie die Generationen vor ihnen. Bevor die Vertriebsschienen Fernsehen und Video funktionierten, drängten auch die älteren Filminteressierten in die Uraufführungskinos, im Zweifel wochenlang. Beispiele wie "Citizen Kane" (Orson Welles, 1940), "Vom Winde verweht" (Victor Fleming, USA 1939) oder Ingmar Bergmans "Das Schweigen" (Schweden 1963) machen diesen Vorgang erinnerlich.

\section{5 .}

Mit dieser aktuellen Neigung zum brandneuen Kinofilm sind die Jüngeren unmittelbarer über Filmstoffe, Trends, Personen, Themen, Probleme orientiert, die in der filmisch verdichteten Form Gesprächsangebote sind (deren Aktualität allerdings auch wieder nicht der Tageslage entspricht, da das Industrieprodukt Film einen langen planerischen und produktionellen Vorlauf braucht). Entscheidend ist, was im Kino gesucht wird, was man zu finden erwartet und auch findet. Das liegt letztlich weniger auf dem Gebiet der Informationserweiterung. Der jüngere Kinogänger will möglichst umfassend bewegt und beeindruckt werden. $\mathrm{Ob}$ durch vorherige Reklame oder Kritik aufmerksam gemacht (wobei das Kino nach wie vor durch Mundpropaganda, durch Kinogänger selbst, am besten funktioniert), wird im Kino ein tiefgreifendes Erlebnis wo nicht gewollt, so doch mit dem Lösen der Kinokarte zugelassen. Die Emotionalität des Kinos stand seit Beginn außer Zweifel. Daß ihr jedoch in diesen Räumen eine solche Wirkungsbreite zukommt, war und ist immer noch neu. Im traditionellen Theater wurde weniger geweint und konnte auch weniger geweint werden als 
im gängigen Kino; beide Räume mit ihren letztlich extrem unterschiedlichen Angeboten funktionieren anders. ${ }^{20}$ Dennoch drängen sich immer wieder Erinnerungen an die klassischen Theaterdramaturgien von Aristoteles bis Lessing und an die damit verbundene, in der gegenwärtigen Wirkungsdebatte um den Film v.a. im Fernsehen immer wieder strapazierte Katharsis-Thematik auf. Befreit das Gesehene durch Furcht, durch Schrecken, durch Gelächter? Ohne in diese fruchtlose Dauerdebatte eingreifen zu wollen, kann jedenfalls dem Gelächter versichert werden, daß es im Kino einen mitunter enorm befreienden Ort besitzt. Zumindest liefert das Kinogeschehen aber Ablenkung. Es konzentriert durch die Erzählungen ganz anderer Schicksale, deren Hintergründe in aller Regel wiedererkennbare Milieus gar nicht so exotischen Zuschnitts sind, die Aufmerksamkeit auf mögliche Folgerungen für eigene Einstellungen.

6.

Der Film spricht die Neugier an, ohne die er gar nicht funktionieren würde; mit ihrem Funktionieren kann er aber in aller Regel rechnen, weil er auf ein vergleichsweise frisches und unvoreingenommen-offenes Bewußtsein und Urteilsvermögen stößt: Welche Schicksale erlebt derjenige dort, welche Gefühle und Erfahrungen machen ihn aus, welche Situation ähnelt dann sogar der eigenen? Letztlich ist die Frage des (jugendlichen) Kinobesuchers, was das Leben so oder so auf dem Wege von Botschaften in Form von Erfahrungen anderer für ihn bereithält, denen er konzentriert zu folgen bereit ist, zunächst durch

20 Aufschlußreich sind Bemühungen um die Erklärung der Emotionalität und Wirkung des Kinofilms wie etwa diejenige von Thomas Mann (1928), der seine grundsätzliche Differenz zwischen Kunst und Leben auch auf den Film projiziert: "... Was mich betrifft, so verachte ich (den Film) auch, aber ich liebe ihn. Er ist nicht Kunst, er ist Leben und Wirklichkeit, und seine Wirkungen sind in ihrer bewegten Stummheit, krud sensationell im Vergleich mit den geistigen Wirkungen der Kunst: es sind die Wirkungen, die Leben und Wirklichkeit auf den unbeteiligten Zuschauer üben, besänftigt durch die Bequemlichkeit der Umstände und das Bewußtsein des gestellten Schauspiels, verstärkt und aufgehöht durch Musik. Sagen Sie mir doch, warum man im Cinema jeden Augenblick weint oder vielmehr heult wie ein Dienstmädchen! Wir waren neulich alle bei der Erstaufführung der ,Großen Parade', auch Olaf Gulbransson, dem wir am Ausgang begegneten. Der lustige, muskulöse Eskimo war tränenüberschwemmt. "Ich habe mich noch nicht abgetrocknet", sagte er entschuldigend, und wir standen noch lange mit feuchten Augen in einfältiger Gelöstheit beieinander. Ist das die Verfassung, in der man von einem Kunstwerk scheidet, einer Malerei den Rücken wendet, ein Buch aus der Hand legt, ein Theater verläßt? ..." (Zit. bei Kaes, Anton (Hg.), Kino-Debatte. Texte zum Verhältnis von Literatur und Film 1909 - 1929, München und Tübingen 1978, 164f). 
Wahrnehmung, später durch Nachahmung.,21 Vorher allerdings sind zunächst und meist ausschließlich die emotionalen Dimensionen der präsentierten Geschichten gefragt: Sehnsucht, Mitleiden, Schmerz, Erleichterung, Solidaritätsgefühle, Identifikationsneigung u.ä.m.

Gefühl und mentales Wahrnehmungsvermögen sind im Kino deswegen von besonderer Erreichbarkeit und Erregbarkeit, weil der Kinoraum eine außerordentliche Konzentrationsmöglichkeit bietet. Die Gerichtetheit der Aufmerksamkeit aller auf die eine Geschichte, der zu folgen die Fixierung und Ausrichtung der Sitze zwingend nahelegt, unterscheidet sich von den Sehgewohnheiten vor dem Fernsehgerät fundamental, weil dort potentielle Ablenkungen zuhauf mitgeliefert werden. Die Möglichkeit konzentrierter Bildwahrnehmung, oft Dimensionen quasi-magischen Gebanntseins, einer Art Andächtigkeit angenähert, steht in direkter Korrespondenz zur Faszination des Mediums. Die Wahrnehmung im Kino hat deshalb eine so unverwechselbare Intensität, weil sie durch die alle Sinne fesselnde Suggestivkraft eine einzigartige Tiefendimension beim Zuschauer erreicht. Nicht ist die von manchen befürchtete Selbstaufgabe eigener Innenwelten, nicht die Selbstvergessenheit Gefahr dieses Erlebnisses. Vielmehr ermöglicht die konzentrierte Wahrnehmung filmischen Erzählens auch die Chance einer gesteigerten Individuation im Hinblick auf die vorgegebenen Bildmuster.

\section{7.}

Eine besondere Chance dieser Art erkenntnissteigernder ästhetischer Wahrnehmung für die eigene Persönlichkeit liegt klassischerweise auch und gerade im Kino in der Möglichkeit gemeinsamen Erlebens. Angesichts der angebotenen Lebensbilder hilft die gemeinschaftliche und dadurch möglicherweise dialogische Rezeption bei der Verdichtung des Wahrgenommenen, bei Verifikation, Identifikation, aber auch bei Verweigerung und Distanzierung. Film im Kino in Gemeinschaft zu erleben, macht in unserer modernen Gesellschaft vermutlich mehr Effekt für den emotionalen Haushalt jüngerer Zuschauer aus als manches systematische pädagogische Angebot. Diese Dimension des Kinoerlebens unterschätzen diejenigen gern, die dem wochenendlichen Kinobesuch von Freund und Freundin, Schüler und Schülerin etc. eher die als geradezu zwingend angesehene erotische Annäherung als wichtigste Funktion zusprechen. Das Gemeinschaftserlebnis des im Kino wahrgenommenen Films ist allerdings auch nicht das einzig denkbare oder adäquate Verhaltensmuster. Film als Massenkommunikationsmittel meint sicherlich nicht eine notwendige Gemeinsamkeit in der Wahrnehmung, sondern die Dimension der Gleichzeitigkeit bei der Wahrnehmung

21 Dieser schlichte Zusammmenhang ist die Grundlage für Jugendschutzüberlegungen seit den Anfängen des Kinofilms. 
durch viele. Das gemeinsame Erleben vor allem auch in einem Raum mit vielen fremden Menschen, in der Anonymität der Zufälligkeit des gleichzeitigen Eintrittskartenerwerbs verschafft allerdings auch eine sich beiläufig ergebene Gruppenatmosphäre, die sich in gleichgerichteten oder auch - was dann nicht selten besonders amüsant ist - sehr unterschiedlichen Gefühlsbekundungen äußert. Die individuelle Wahrnehmung allerdings ist zunächst Voraussetzung aller und "darf" hinreichend sein für den, der gerade wegen der hohen Erregtheit bzw. Berührtheit durch einen bestimmten Film alles andere beabsichtigt als den anschließend ernüchternd-rationalen Diskurs mit anderen.

\section{8.}

Die angebotenen Kinogeschichten waren seit Beginn des Mediums Film vom Ehrgeiz bewegt, möglichst große Geschichten zu sein. Die inhaltliche Dimension wies und weist über den Alltag schierer Wiedererkennbarkeit durch den ersten Blick des Zuschauers in aller Regel weit hinaus. Kinofilm will immer Bewußtseinserweiterung, hat eine hohe Ambition, Wirklichkeit zu transzendieren, einen Kosmos vorzuführen von möglichst großer Umfänglichkeit, sei er

- geographisch-physikalisch angelegt: Stanley Kubrick: 2001 - Odyssee im Weltraum, USA 1968; George Lucas, Krieg der Sterne, USA 1977; Steven Spielberg, Unheimliche Begegnung der dritten Art, USA 1977; Irvin Kershner, Das Imperium schlägt zurück, USA 1979; Ridley Scott, Alien, USA 1979; Steven Spielberg, E.T. - Der Außerirdische, USA 1982 und andere vergleichbare oder auch trivialere ScienceFiction-Filme;

- historisch orientiert: D. W. Griffith, Intolerance, USA 1916; Sergej Eisenstein, Panzerkreuzer Potemkin, UdSSR 1925; Iwan der Schreckliche, UdSSR 1944/46; Mervyn LeRoy, Quo vadis?, USA 1951; King Vidor, Krieg und Frieden, USA/Italien 1956; William Wyler, Ben Hur, USA 1959; David Lean, Lawrence von Arabien, GB 1962; Sergej Bondartschuk, Krieg und Frieden, UdSSR 1966/67; Francis Ford Coppola, Apocalypse now, USA 1979; Richard Attenborough, Gandhi, GB 1981/82; Jean-Jacques Annaud, Der Name der Rose, Bundesrepublik/Frankreich/Italien 1986; Bernardo Bertolucci, Der letzte Kaiser, GB 1986/87; Steven Spielberg, Schindlers Liste, USA 1993;

- im kriminalistischen oder phantastischen Spannungsbereich angesiedelt: Friedrich Wilhelm Murnau, Nosferatu - Eine Symphonie des Grauens, D 1992; James Whale, Frankenstein, USA 1931; John Huston, Die Spur des Falken, USA 1941; Howard Hawks, Tote schlafen fest, USA 1946; Carol Reed, Der dritte Mann, GB 1949; Alfred Hitchcock, Das Fenster zum Hof, USA 1954; Alexander Mackendrick, Ladykillers, GB 1955; Billy Wilder, Zeugin der Anklage, USA 1957; Alfred Hitchcock, Vertigo - Aus dem Reich der Toten, USA 1958; Psycho, USA 1960; Die Vögel, USA 1962, Die 
James-Bond-Filme (007) seit 1962; Jean-Pierre Melville, Der eiskalte Engel, Frankreich 1967; Arthur Penn, Bonnie und Clyde, USA 1967; Don Siegel, Dirty Harry, USA 1971; Roman Polanski, Chinatown, USA 1974; Peter Weir, Der einzige Zeuge, USA 1985; Jonathan Demme, Das Schweigen der Lämmer, USA 1990;

- emotional auslotende Beziehungsgeschichte oder gesellschaftliches Drama: Josef von Sternberg, Der blaue Engel, D 1930; George Cukor, Die Kameliendame, USA 1936; Victor Fleming, Vom Winde verweht, USA 1939; Michael Curtiz, Casablanca, USA 1942; Marcel Carné, Kinder des Olymp, Frankreich 1943; Charles Vidor, Gilda, USA 1946; Billy Wilder, Boulevard der Dämmerung, USA 1950; William Wyler, Ein Herz und eine Krone, USA 1953; Federico Fellini, La Strada, Italien 1954; François Truffaut, Jules und Jim, Frankreich 1961; Claude Lelouche, Ein Mann und eine Frau, Frankreich 1966; Bernardo Bertolucci, Der letzte Tango in Paris, Italien/Frankreich 1972; Woody Allen, Manhattan, USA 1978; Sidney Pollack, Jenseits von Afrika, USA 1985; Adrian Lyne, Eine verhängnisvolle Affäre, USA 1987; Barry Levinson, Rain Man, USA 1988; Garry Marshall, Pretty Woman, USA 1989; Jane Campion, Das Piano, Australien 1992; Jonathan Demme, Philadelphia, USA 1993;

- dem Gelächter gewidmet: die Filme von Charlie Chaplin von Anfang an; die Filme der anderen großen Filmkomiker wie Harold Lloyd, Buster Keaton, "Fatty" Arbuckle, der Marx-Brothers, Laurel \& Hardy u.v.a.m.; Ernst Lubitsch, Sein oder Nichtsein, USA 1942; Frank Capra, Arsen und Spitzenhäubchen, USA 1944; Robert Hamer, Adel verpflichtet, GB 1949; Julien Duvivier, Don Camillo und Peppone, Frankreich/Italien 1952; Jacques Tati, Die Ferien der Herrn Hulot, Frankreich 1953; Alexander Mackendrick, Ladykillers, GB 1955; Helmut Käutner, Der Hauptmann von Köpenick, Bundesrepublik 1956; Billy Wilder, Manche mögens heiß, USA 1959; Das Appartement, USA 1959; Blake Edwards, Frühstück bei Tiffany, USA 1960; Billy Wilder, Das Mädchen Irma la Douce, USA 1962; Roman Polanski, Tanz der Vampire, GB 1966; George Roy Hill, Der Clou, USA 1973; Terry Jones, Monty Python's - Das Leben des Brian, GB 1979; Sidney Pollack, Tootsie, USA 1982; Robert Zemeckis, Zurück in die Zukunft, USA 1984; Doris Dörrie, Männer, Bundesrepublik 1985; Peter Faiman, Crocodile Dundee, USA/Australien 1986; Charles Crichton, Ein Fisch namens Wanda, USA 1987; Rob Reiner, Harry und Sally, USA 1989; Chris Columbus, Kevin - Allein zu Haus, USA 1990;

- auf der Suche nach dem Abenteuerlichen: M.C. Cooper/E.B. Schoedsack, King Kong und die weiße Frau, USA 1933; John Ford, Ringo (Stage Coach), USA 1939; John Huston, African Queen, USA 1951; Henri-Georges Clouzot, Lohn der Angst, Frankreich 1952; Fred Zinnemann, Zwölf Uhr mittags, USA 1952; William Wyler, Weites Land, USA 1958; Jean-Luc Godard, Außer Atem, Frankreich 1959; Sergio Leone, Spiel mir das Lied vom Tod, Italien 1968; Dennis Hopper, Easy Rider, USA 1969; Steven Spielberg, Der weiße Hai, USA 1974; Sylvester Stallone, Rocky, USA 1976; Steven Spielberg, 
Jäger des verlorenen Schatzes, USA 1980; Sylvester Stallone, Rambo, USA 1982; Kevin Costner, Der mit dem Wolf tanzt, USA 1990; Steven Spielberg, Jurassic Park, USA 1993;

- an ungewöhnlicher inhaltlicher Fragestellung interessiert: Sidney Lumet, Die zwölf Geschworenen, USA 1957; Federico Fellini, Das süße Leben, Italien 1959; Michelangelo Antonioni, Blow up, GB 1966; Stanley Kubrick, Uhrwerk Orange, GB 1970/71; Milos Forman, Einer flog über das Kuckucksnest, USA 1975; Martin Scorcese, Taxi Driver, USA 1975;

- auf Adaption von Literatur, Musik und Tanz ausgerichtet: Victor Fleming, Das zauberhafte Land, USA 1939; S. Donen/G. Kelly, Du sollst mein Glücksstern sein (Singin' in the rain), USA 1952; Elia Kazan, Jenseits von Eden, USA 1955; Robert Wise/Jerome Robbins, West Side Story, USA 1960; David Lean, Doktor Schiwago, USA 1965; Luchino Visconti, Tod in Venedig, Italien 1970; Bob Fosse, Cabaret, USA 1971; Jim Sharman, The Rocky Horror Picture Show, USA 1974; Robert Altman, Nashville, USA 1975; Volker Schlöndorff, Die verlorene Ehre der Katharina Blum, Bundesrepublik 1975; Die Blechtrommel, Bundesrepublik 1978; Adrian Lyne, Flashdance, USA 1982; Carlos Saura, Carmen, Spanien 1983; Milos Forman, Amadeus, USA 1984; Emile Ardolino, Dirty Dancing, USA 1987;

- an Ausweitung des Seherlebnisses orientiert: Walt Disney, Bambi, USA 1942; Alain Resnais, Letztes Jahr in Marienbad, Frankreich 1960; François Truffaut, Die amerikanische Nacht, Frankreich 1972; Stanley Kubrick, Barry Lyndon, GB 1973/75; Ivan Reitman, Ghostbusters, USA 1984; Steven Soderbergh, Sex, Lügen und Video, USA 1989; Garry Trousdale/Kirk Wise, Die Schöne und das Biest, USA 1991; John Musker/Ron Clements, Aladdin, USA 1992;

- Geheimnissen und Bedrohungen menschlichen Zusammenlebens auf der Spur: Orson Welles, Citizen Kane, USA 1940; Roman Polanski, Rosemaries Baby, USA 1967; William Friedkin, Der Exorzist, USA 1973; James Bridges, Das Chinasyndrom, USA 1978; David Lynch, Blue Velvet, USA 1986; Peter Weir, Der Club der toten Dichter, USA 1988;

- religiös-spirituell fragend: Cecil B. de Mille, Der König der Könige, USA 1926/27, Nicholas Ray, König der Könige, USA 1960; George Stevens, Die größte Geschichte aller Zeiten, USA 1963; Ingmar Bergman, Das Schweigen, Schweden 1963; Pier Paolo Pasolini, Das erste Evangelium - Matthäus, Italien 1964; Norman Jewinson, Jesus Christ Superstar, USA 1972; Andrej Tarkowskij, Solaris, UdSSR 1972; Franco Zeffirelli, Jesus von Nazareth, Italien/GB 1976; Andrej Tarkowskij, Der Stalker, UdSSR 1979; Jean-Luc Godard, Maria und Joseph, Frankreich/Schweiz 1984; Andrej Tarkowskij, Opfer, Schweden/Frankreich 1985; Denys Arcand, Jesus von Montreal, Kanada 1989. 
9.

Ein derartiger Durchgang durch einhundert Jahre Filmgeschichte, der nur sog. "große" Filme berücksichtigt, deren Bedeutung neben ihrer inhaltlichen und formalen Überdurchschnittlichkeit und Relevanz von ihrem in aller Regel außerordentlichen Erfolg beim Publikum (weltweit) bestimmt ist ${ }^{22}$, macht deutlich, welche weitgespannte inhaltliche und formale Dimension der internationale Film entwickelt hat. ${ }^{23}$ Der Film hat universellen Anspruch. Seine Bilderwelten bilden einen umfassenden Kosmos. Dessen Dimensionierung kam schon in der Zeit des gezielten Baus von Kinos - in den $20 \mathrm{er}$ und 30er Jahren -, als das Medium seine feste Etablierung erlebte, in Kinonamen wie "Universum" zum Ausdruck. Wie aus den genannten Filmen und deren weitgespannter Genrezuordnung zu schließen ist, hat sich der Film den universellen Anspruch erhalten und schreibt ihn fort.

Die Bezeichnungen für die Vorführstätten des Kinofilms, für die neueren Kinobauten, sind nüchterner geworden, funktionaler. Nach den innerstädtischen "Filmpalästen" der 20er Jahre in Deutschland und Mitteleuropa und den "Kino-Kathedralen" der Zeit in den USA ${ }^{24}$ entstehen - nachdem der zwischenzeitliche Boom auch Kleinstädte und Dörfer erreicht hatte - inzwischen immer häufiger monumentale Gründungen auf stadtperipheren Freianlagen, die "Cineplex" oder "Multiplex", die unter ihren Dächern zwischen 10 und 20 Kinos beherbergen, in denen das gesamte aktuelle Angebot zumindest gängigen Zuschnitts gesichtet werden kann. Diese Film- und FreizeitZentren haben erneut universellen Anspruch, der nicht ohne gigantomanische Züge ist. Das Kölner Multiplex-Kino nennt sich nicht ohne Ambition "Cinedom", um die architektonische Dimensionierung und den universalen inhaltlichen Anspruch, welche den Domkirchen eignen, nun für die Filmwelt anzumelden.

22 Wobei auch Titel genannt wurden, die entgegen cineastischer Einschätzung konsequenterweise in die Betrachtung einbezogen werden mußten, da sie trotz erheblicher Fragwürdigkeit (im Hinblick auf Gewalt-Darstellung z.B. Stallones „Rambo") oder populistischer Kultivierung von Oberflächenspannung (Spielbergs "Jäger des verlorenen Schatzes") zu den erfolgreichsten bzw. attraktivsten Produkten der Filmgeschichte gehören.

23 Dabei ist wegen der Verbreitungs- und Erfolgskomponente der amerikanische Film - wie zu sehen war - besonders stark berücksichtigt worden: Er dominiert die Märkte. Andere Länder und Kontinente wie Afrika, Indien, Südamerika, Polen, Ungarn, die CSSR, Schweiz, Belgien u.v.a.m. blicken auf ein nicht unerhebliches Filmschaffen zurück.

24 Den Begriff „Movie Cathedral” verwendet z.B. Ronald Holloway, Beyond the image. Approaches to the religious dimension in the cinema, Geneva $1977,75-104$. 
In den Kino-Kathedralen wird nicht meditativ-beschaulich die eigene Innenwelt mit dem Heiligen in Verbindung gebracht. Dazu sind die angebotenen Stoffe und Themen $\mathrm{zu}$ eigendynamisch, $\mathrm{zu}$ spektakulär, trivial bis vulgär. Aber die Möglichkeit der konzentrierten Begegnung mit dem Unerhörten, Erhebenden und Erhabenen gibt dem Kino doch eine eigene und außergewöhnliche Wertigkeit für die persönliche Erlebniswelt. Im Kino des "multiplexen" Programms finden sich eben auch Sinnangebote, Problemdarstellungen, Beziehungsgeschichten, Lösungsmuster aufgrund erzählter Geschichten von anderen Menschen, die geeignet sind, eigenes Verhalten zu kanalisieren oder gänzlich zu modifizieren. Wenn dabei auch sog. „kleine“ Geschichten mitgeliefert werden, so ist dies eine immer wieder stattfindende Ergänzung des Mainstream-Angebots der besonders attraktiven Titel und Themen, die in der Werbung besonders hofiert werden. Der Markt des Kinofilmangebots hat eine nicht unerhebliche magische Dimension: Daß hier viele Geschichten erzählt werden - natürlich zwischen Popcorn, Cola, Bier und Pizza -, ist die Gewähr für eine Orientierungsmöglichkeit an den Erfahrungen anderer, an deren Träumen, Hoffnungen, Sehnsüchten, deren Traurigkeiten und Befürchtungen, deren Leidenschaft und Lust, angeboten in attraktiver Verpackung eines komplexen und raffiniert hergestellten Industrieprodukts. Diese Offerten sinnstiftender und problemlösender Geschichten immer wieder wahrzunehmen, sie abzuholen und zu nutzen, drängen Abend für Abend, vor allem aber an den Wochenenden die Angehörigen der Jahrgänge bis 30 in die Kinosäle. Der Vorgang hat eine kultische Dimension. Unausgesprochen erwartet man in der wohligen Geborgenheit des Kinodunkels ein Stück Erlösung von Irritation, Frust, Einsamkeit, Lieblosigkeit, Scheitern, wenn auch nur für wenige Stunden.

Diese „Erlösungserwartung“ kann auf berufene und würdige wie auch auf fatale und verwirrende Angebote stoßen. Der Vorgang als solcher aber ist von subtiler Bedeutung, daß nämlich auf diesem diffusen Markt magischer Versprechungen Lösungen gesucht werden, was eine allgemeine Freizeitdiskussion unter dem Sammelbegriff Unterhaltungsbedürfnis abzulegen gewohnt ist.

Nach 100 Jahren Kinoentwicklung, an der auch die katholische Kirche zunehmend Anteil genommen hat ${ }^{25}$, sollte die Kirche wahrnehmen, daß es das Kino in alter Vitalität gibt als einen höchst lebendigen

25 Durch Gründung einer eigenen internationalen katholischen Filmorganisation (OCIC) 1928, durch zahlreiche kirchenamtliche Äußerungen, durch mannigfaltige filmpädagogische Bemühungen sowie nicht zuletzt durch eine ausgefeilte und international vielfältige Filmpublizistik, die sich vor allem mit dem jeweiligen einzelnen Filmprodukt beschäftigt (Beispiele: film-dienst in Deutschland, Zoom in der Schweiz, multimedia in Österreich, Fiches du Cinema in Frankreich etc.). 
Marktplatz von Gefühlen, Lebensbildern, Gedanken und Entwürfen vor allem der jüngeren Generation. Deren Angehörige drängen nicht in die Kirchen als den berufeneren Stätten der Besinnung und Andacht, um sich Anrufen zu öffnen. Es bleibt wichtig, kirchlicherseits die Inhalte zu kennen, die Trends mitzubekommen, die Probleme und Diskussionen, die von Filmen ausgehen, in einen Dialog über Film einzubringen. Film als das technisch-kulturelle Faszinosum des Jahrhunderts darf nicht lediglich als explizites Medium der Verkündigung interessieren. Denn auf diesem Sektor liegen seine besten Fähigkeiten durchaus nicht, was das Gros bekannter und unbekannter Bibelverfilmungen zeigt. Der Film muß zuallererst interessieren als das, was er immer war und immer wieder sein kann, nämlich ein faszinierendes Zeugnis einer atemberaubend lebendigen Welt, auf die einzulassen sich lohnt.

SUMMARY: Market and Magic, Communication and Cult. Cinema as a Place for Sensation

Movies are a fascinating evidence for a breathtaking vivid world. Cinema as a place for sensation gives opportunity to feel emotions, to be distraced, curious or fascinated and to share experiences. The event of watching a movie in a cinema has a ritual dimension. The audience expects a relief from the distress of everyday life in the cosy security of the dark auditorium. The gigantic features of cinema architecture meet this subjective need for release. The magnitude of the buildings of the last few years corresponds to the claim for universal significance of film culture. The Catholic Church has increasingly participated in the development of film culture. It remains important to be aware of trends and to partake in the discourse about film.

RÉSUMÉ: Marché et magie, communication et culte. Le cinéma en tant qu'espace d'évènements

Les films présentent des témoignages fascinants $d$ 'un monde vivant et palpitant. Le cinéma en tant qu'espace d'évènements offre de la place à l'émotion, la distraction, la curiosité, la fascination et l'aventure partagée. Le processus de la vue $d^{\prime} u n$ film au cinéma a une dimension culturelle. Dans l'enceinte sécurisante et agréable des salles obscures, les spectateurs attendent une certaine délivrance des déceptions du quotidien. A ce besoin subjectif de libération correspondent les traits de gigantisme de l'architecture des cinémas. Les dimensions des cinémas construits ces dernières années correspondent à la prétention universelle en ce qui concerne le contenu de la culture cinématographique. A l'avenir, il sera important de connaître les tendances et de participer activement aux discussions se rapportant aux films.

RESUMEN: Mercado y magia, comunicación y culto. Referente al cine como lugar donde ocurren acontecimientos

Las películas se nos presentan como fascinantes testimonios de un mundo increíblemente vivo. El cine como un espacio de acontecimientos abre espacios para la emocionalidad, la distracción, la curiosidad, la fascincación y 
para la experiencia de una vivencia conjunta. La experiencia de ver películas en el cine tiene una dimensión de culto. Los espectadores esperan en el agradable arropamiento de la oscuridad de un cine una liberación de las decepciones de la vida diaria. A esta subjetica necesidad de liberación corresponden los gigantescos dimensiones de la arquitectura de los cines. Las dimensiones de las construcciones de cines en los últimos años corresponden al contenido universal de la cultura del film. La iglesia católica ha hecho un aporte creciente al desarrollo de esta cultura del film. A futuro sigue siendo importante el conocer de las tendencias y participar activamente en el diálogo sobre películas. 